\title{
Reduced TGF- $\beta$ Expression and CD206-Positive Resident Macrophages in the Intervertebral Discs of Aged Mice
}

\author{
Yuji Yokozeki, ${ }^{1}$ Ayumu Kawakubo, ${ }^{1}$ Masayuki Miyagi ${ }^{(D)},{ }^{1}$ Akiyoshi Kuroda, ${ }^{1}$ \\ Hiroyuki Sekiguchi, ${ }^{2}$ Gen Inoue $\mathbb{D}^{1},{ }^{1}$ Masashi Takaso, ${ }^{1}$ and Kentaro Uchida ${ }^{10}{ }^{1,2}$ \\ ${ }^{1}$ Department of Orthopedic Surgery, Kitasato University School of Medicine, 1-15-1 Minami-ku Kitasato, Sagamihara City, \\ Kanagawa 252-0374, Japan \\ ${ }^{2}$ Shonan University of Medical Sciences Research Institute, Nishikubo 500, Chigasaki City, Kanagawa 253-0083, Japan
}

Correspondence should be addressed to Kentaro Uchida; kuchida@med.kitasato-u.ac.jp

Received 5 April 2021; Accepted 3 July 2021; Published 12 July 2021

Academic Editor: Kazim Husain

Copyright (C) 2021 Yuji Yokozeki et al. This is an open access article distributed under the Creative Commons Attribution License, which permits unrestricted use, distribution, and reproduction in any medium, provided the original work is properly cited.

\begin{abstract}
Age is a key factor in intervertebral disc (IVD) degeneration; however, the changes that occur in IVDs with age are not fully understood. Tissue-resident macrophages are critical for tissue homeostasis and are regulated by transforming growth factor(TGF-) $\beta$. We examined changes in the proportion of resident macrophages in young versus aged mice and the role of TGF- $\beta$ in regulating resident macrophages in IVDs. IVDs were harvested from 4-month (young) and 18-month-old (aged) C57BL/6J mice. The proportion of macrophages in IVDs was determined using flow cytometry ( $n=5$ for each time point) and the expression of $C d 11 b, C d 206$, and Tgfb genes, which encode CD11b, CD206, and TGF- $\beta$ protein, respectively, using real-time PCR. To study the role of TGF- $\beta$ in the polarization of resident macrophages, resident macrophages isolated from IVDs from young and aged mice were treated with recombinant TGF- $\beta$ with and without a TGF- $\beta$ inhibitor (SB431542). Additionally, SB431542 was intraperitoneally injected into young and aged mice, and Cd206 expression was examined using real-time PCR ( $n=10$ for each time point). The proportion of CD11b+ and CD11b+CD206+ cells was significantly reduced in aged versus young mice, as was $C d 11 b, C d 206$, and Tgfb expression. TGF- $\beta /$ IL10 stimulation significantly increased the expression of $C d 206$, an M2 macrophage marker, in disc macrophages from both young and aged mice. Meanwhile, administration of a TGF- $\beta$ inhibitor significantly reduced Cd206 expression compared to vehicle control in both groups. Conclusion. Resident macrophages decrease with age in IVDs, which may be associated with the concomitant decrease in TGF- $\beta$. Our findings provide new insight into the mechanisms of age-related IVD pathology.
\end{abstract}

\section{Introduction}

Intervertebral disc (IVD) degeneration is frequently linked to low back pain $[1,2]$, and age is a key factor in IVD degeneration. Evidence suggests that several factors, including altered extracellular matrix components, anabolic/catabolic balance, cell senescence, and inflammation, are associated with aging-related IVD degeneration [3-6]. However, the changes that occur in IVDs with age are not fully understood.

Various kinds of macrophages, including resident macrophages and recruited macrophages, are present in degenerated IVDs [7-10]. Recruited macrophages differentiate into proinflammatory phenotype M1 macrophages, which are thought to induce inflammation in IVDs [7]. In contrast, tissue-specific macrophages, known as resident macrophages, exist in a variety of tissues [10-14]. Recent studies have demonstrated that resident macrophages exist in mouse and human IVDs $[9,10,13]$. Resident macrophages exhibit M2 phenotype and play an important role in promoting regeneration, inflammation resolution, and remodeling [15]. However, a previous study reported that resident macrophages in the heart decrease and show declining selfrenewal activity with age [16]. Age-related reductions in resident macrophages in IVDs may form part of the mechanism 
underlying age-related IVD degeneration. However, the factors causing age-related decreases in resident macrophages remain to be determined.

Transforming growth factor-beta (TGF- $\beta$ ) signaling plays important roles in a number of cellular processes, including cell proliferation, migration, differentiation, and apoptosis, in different cell types, and is associated with aging-related pathology [17]. TGF- $\beta$ and its receptors have been observed in nucleus pulposus and annulus fibrosus cells in young mice; the expressions of which are reduced with age [18]. Further, recent studies have reported that TGF- $\beta$ promotes the development and homeostasis of resident macrophages in the skin and lung $[19,20]$. We hypothesized that age-related decreases in TGF- $\beta$ may alter resident macrophage populations in IVDs.

Here, we examined changes in the proportion of resident macrophages in young versus aged mice and the role of TGF$\beta$ in regulating resident macrophages in IVDs.

\section{Materials and Methods}

2.1. Animals. The study protocol received approval from the Kitasato Institutional Animal Care Committee (reference number: 2020-089). The study complied with the ARRIVE guidelines for the reporting of animal experiments. All methods were conducted based on the guidelines for the proper conduct of animal experiments by the Science Council of Japan.

Male C57BL/6J (B6) mice aged 4 (young) and 18 (aged) months (30 mice each) were housed in a housing system maintained at $25 \pm 1{ }^{\circ} \mathrm{C}$ and $60 \pm 5 \%$ humidity with a 12:12-hour light: dark cycle throughout the study period.

Coccygeal (Co) 5/6, Co6/7, and Co7/8 IVDs were resected without separating the nucleus pulposus (NP) and annulus fibrosus (AF) and pooled for each measurement.

2.2. Flow Cytometric Analysis. IVDs were harvested from young and aged mice ( $n=5$ each) and digested with $2 \mathrm{mg} / \mathrm{ml}$ collagenase type I solution (Product no. 032-22364, Fujifilm Wako Pure Chemical Corporation, Osaka, Japan) at $37^{\circ} \mathrm{C}$ overnight, before being passed through a nylon mesh filter with pore size $100 \mu \mathrm{m}$. The resulting single-cell suspensions were incubated with PE/Cy7-conjugated anti-CD45 (Clone: 30-F11, Product no. 103114, BioLegend, CA, USA) and APC-Cy7-conjugated anti-CD11b (Clone: M1/70, Product no. 101226, BioLegend) antibodies for $40 \mathrm{~min}$ at $4^{\circ} \mathrm{C}$ and subsequently treated with fixation/permeabilization solution (Product no. 420801, BioLegend). The cells were then incubated with APC-conjugated CD206 antibody (Clone: C068C2, Product no. 141708, BioLegend) for $30 \mathrm{~min}$ at $4^{\circ} \mathrm{C}$ and washed twice in wash buffer. The labeled cells were subjected to flow cytometry, in which 50,000 total events were acquired using a BD FACSVerse system (BD Biosciences, San Jose CA, USA), and the results were analyzed using FlowJo v10.7 $7^{\mathrm{TM}}$ (Tree Star, Ashland OR, USA). Negative gates were determined using isotype control.

2.3. Real-Time PCR Analysis. IVDs were harvested from young and aged mice ( $n=10$ each). Standard TRIzol (Prod- uct no. 15596026, Invitrogen, Carlsbad, CA)/chloroform methods were used to extract total RNA from IVD samples. SuperScript III RT ${ }^{\mathrm{TM}}$ (Product no. 18080085, Invitrogen) was subsequently used to synthesize first-strand cDNA for use in real-time PCR with SYBR ${ }^{\mathrm{TM}}$ Green (Product no. 204054, Qiagen, Valencia CA, USA). We examined the expression of $C d 11 b$ (macrophage marker), Cd206 (M2 maker), and Tgfb genes, which encode CD11b, CD206, and TGF- $\beta$ protein, respectively, based on previous reports [9, 13, 21]. Primers were generated according to our previous report $[9,13,21]$. The delta-delta $\mathrm{Ct}$ method and the housekeeping gene Gapdh were used to determine relative mRNA expression levels of the genes of interest.

2.4. Effect of TGF- $\beta$ Inhibitor on Resident Macrophages In Vitro. Following collagenase digestion, IVD-derived cells obtained from young and aged mice, as described above $(n=5)$, were incubated with biotin-conjugated anti-CD11b (Clone: M1/70, Product no. 101204, BioLegend) antibodies for $30 \mathrm{~min}$ at $4^{\circ} \mathrm{C}$. After washing twice with PBS at $300 \times \mathrm{g}$ at $4^{\circ} \mathrm{C}$, IVD cells were reacted with streptavidin-magnetic particles (Product no. 557812, BD Biosciences, San Diego, CA, USA) for $30 \mathrm{~min}$ at $4^{\circ} \mathrm{C}$, before incubating on ice for $8 \mathrm{~min}$ in an IMag separation system (Product no. 552311, BD Biosciences). After aspirating the unbounded cells (CD11b-negative cells), the tube was removed from the magnetic support to obtain the CD11b-positive cells, and $5 \mathrm{ml}$ of culture medium was added. The positive fraction was washed twice with culture medium ( $\alpha$-MEM with $10 \%$ fetal bovine serum) at $300 \times \mathrm{g}$ at $4^{\circ} \mathrm{C}$, before being incubated in culture medium supplemented with $100 \mathrm{ng} / \mathrm{ml}$ recombinant mouse macrophage colony-stimulating factor (M-CSF; Product no. 576406, BioLegend) for 7 days. Following the culture period, macrophages were stimulated with culture medium (vehicle), $10 \mathrm{ng} / \mathrm{ml}$ mouse recombinant TGF- $\beta$ (mrTGF- $\beta$; Product no. 7666-MB-005/CF, R\&D Systems, Minneapolis, MN, USA) + $10 \mathrm{ng} / \mathrm{ml}$ mouse recombinant IL-10 (mrIL-10; Product no. 575806, BioLegend), or mrTGF- $\beta+\mathrm{IL}-10+1 \mu \mathrm{M}$ SB431542 (TGF- $\beta$ inhibitor; Product no. S4317, SigmaAldrich, St Louis, MO, USA) for 24 hours. M2 marker (Cd206) expression was subsequently determined using real-time PCR analysis.

2.5. Effect of TGF- $\beta$ Inhibitor on Cd206 Expression in IVDs. Young and aged C57BL/6J mice were randomly and equally divided into control and treatment groups $(n=10$ each). The control group received an intraperitoneal (IP) injection of $5 \%$ DMSO solution (vehicle), while the treatment group was given an IP injection of $10 \mathrm{mg} / \mathrm{kg}$ SB431542 in 5\% DMSO solution. Dosage was chosen according to the optimal inhibitory effect observed at an IP dose of $10 \mathrm{mg} / \mathrm{kg}$, as reported previously [22, 23]. After 24 hours, IVDs were harvested from young and aged mice and $C d 206$ expression was determined using real-time PCR.

2.6. Statistical Analysis. Distribution of the data was evaluated using the Kolmogorov-Smirnov test. Mann-Whitney $U$ tests were used to evaluate differences between the 4and 18-month groups. Bonferroni multiple comparisons test 


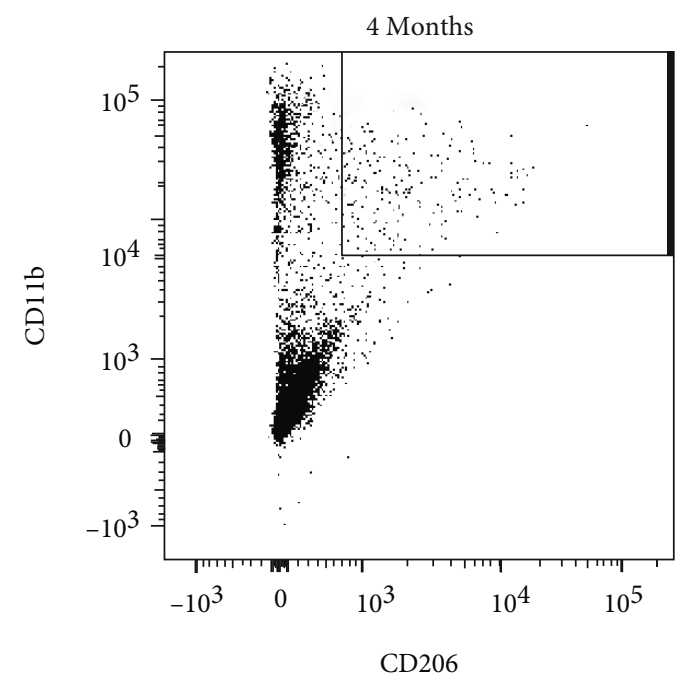

(a)

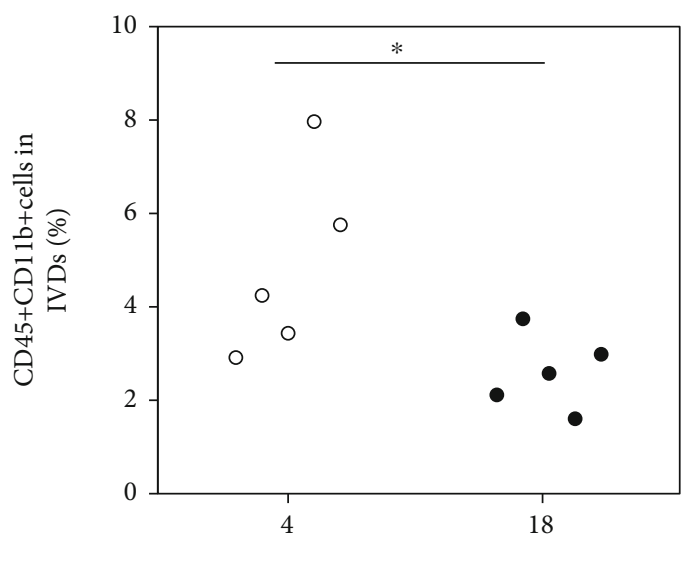

Age (months)

(c)

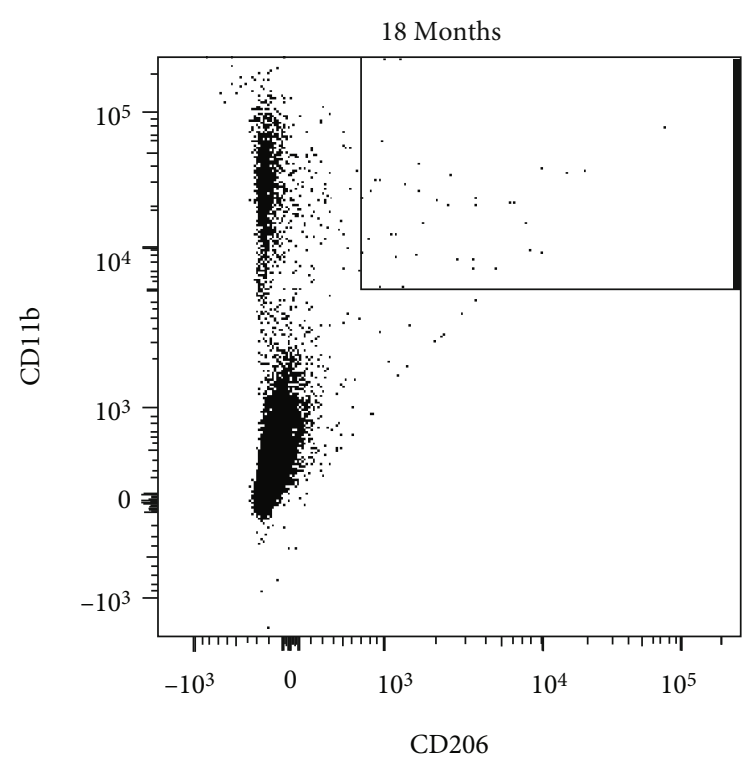

(b)

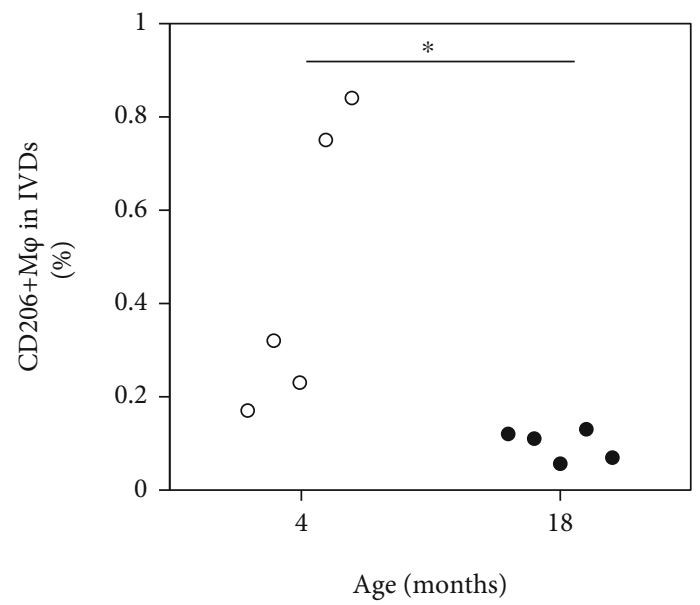

(d)

FIGURE 1: Flow cytometry analysis of the macrophage population in intervertebral discs of young and aged mice. Dot plots showing CD11b+ and CD11b+CD206+ cells in intervertebral discs (IVDs) of (a) young (age 4 months) and (b) aged (age 18 months) mice. The $x$-axis indicates CD206 and the $y$-axis indicates CD11b. (c) Percentage of CD45+ CD11b+cells in IVDs $(n=5)$. (d) Percentage of CD11b+ CD206+ cells in IVDs $(n=5) .{ }^{*} P<0.05$ compared with young mice.

with one-way ANOVA was used to determine differences among the vehicle and TGF- $\beta /$ IL- 10 or TGF- $\beta /$ IL10/SB431542 treatment groups.

\section{Results}

3.1. Proportion of Resident Macrophages and Tgfb Expression in Young and Aged Mice. The proportion of CD11b+ and $\mathrm{CD} 11 \mathrm{~b}+\mathrm{CD} 206+$ cells was significantly reduced in aged versus young mice $(\mathrm{CD} 11 \mathrm{~b}+, P<0.047$; CD11b $+\mathrm{CD} 206+$, $P=0.009$; Figure 1). Consistent with these flow cytometry findings, $C d 11 b$ and $C d 206$ expression was also significantly reduced in aged versus young mice $(C d 11 b, P=0.007$; CD206, $P<0.001$; Figures 2(a) and 2(b)). Likewise, Tgfb expression was significantly reduced in aged versus young mice $(P<0.001$; Figure $2(c))$.
3.2. Effect of TGF- $\beta$ Inhibitor on Cd206 Expression in Resident Macrophage Cell Culture. Expression of the M2 macrophage marker Cd206 was significantly elevated following TGF$\beta /$ IL10 stimulation of macrophages derived from young and aged mice (young, $P=0.030$; aged, $P=0.002$; Figure 3 ). This increase was significantly reversed following exposure to SB431542 (young, $P=0.030$; aged, $P=0.002$; Figure 3). $C d 206$ expression was comparable in macrophages derived from young and aged mice subjected to the same culture conditions.

3.3. Effect of TGF- $\beta$ Inhibitor on Cd206 Expression in IVDs In Vivo. An IP injection of the TGF- $\beta$ inhibitor SB431542 significantly reduced $C d 206$ expression relative to vehicle control in both young and aged mice $(P<0.001$ and $P=0.047$, respectively; Figure 4). 


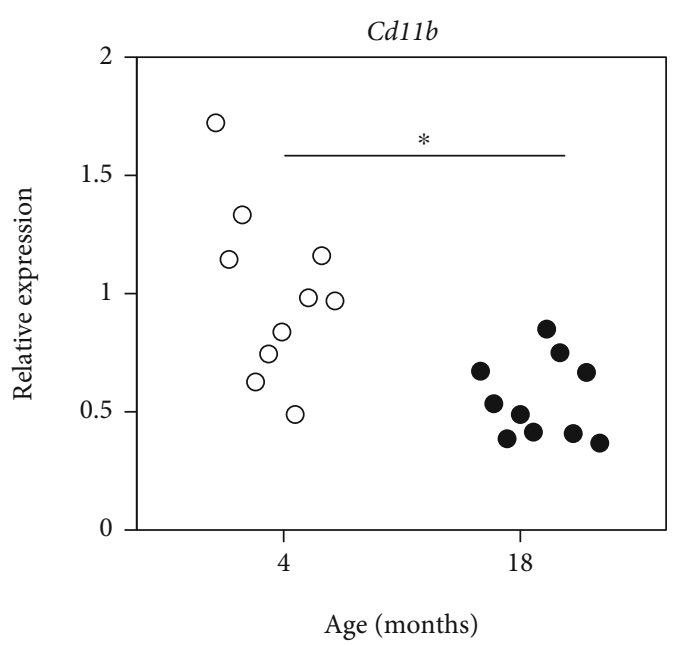

(a)

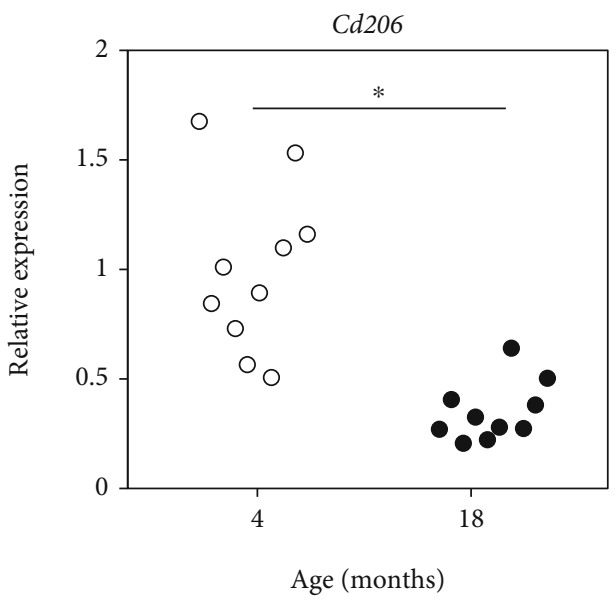

(b)

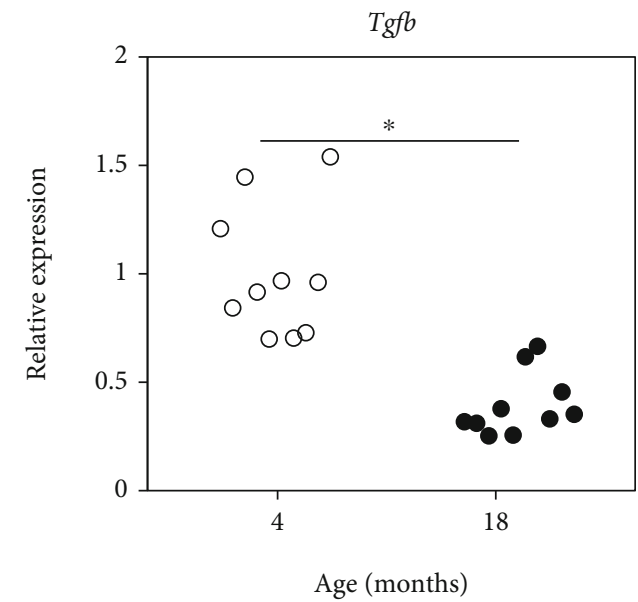

(c)

Figure 2: Expression of $C D 11 b, C d 206$, and Tgfb in intervertebral discs of young and aged mice. Expression of (a) $C d 11 b$, (b) $C d 206$, and (c) $\mathrm{Tg} f \mathrm{~b}$ in intervertebral discs of young (age 4 months) and aged (age 18 months) mice. ${ }^{*} P<0.05$.

\section{Discussion}

Tissue-resident macrophages are critical for tissue homeostasis and immunomodulation. Mice lacking resident macrophages in the epidermis, known as Langerhan cells (LCs), which express IL-10 [24], develop exaggerated contact hypersensitivity. Loss of alveolar macrophages hinders the accumulation of pulmonary surfactants, ultimately causing pulmonary alveolar proteinosis $[12,25,26]$. In our study, we observed a heterogeneous population comprising CD206- and CD206+ macrophages in mouse IVDs, both of which decreased with age. Numerous studies have suggested that CD206+ resident macrophages play an important role in immunosuppression [27, 28]. In allergic skin inflammation, Th2 cytokines induce CD206+ M2 macrophagemediated anti-inflammatory activity [28]. CD206+ M2 macrophages also show anti-inflammatory activity during endotoxemic lung injury through inhibiting the production of a number of proinflammatory cytokines [27]. The role of resident macrophages in IVDs, however, remains unclear. We hypothesized that the age-related reduction in resident macrophages may be associated with age-related IVD degeneration.

Previous studies have shown that, in addition to being self-regulatory, TGF- $\beta$ regulates resident macrophages in adult mice $[19,20]$. TGF- $\beta$ regulates LCs, with the number of LCs shown to decrease in the absence of TGF- $\beta$ [20]. Treatment of $R 26^{C r e E R} T g f b r 2^{f l f l}$ mice with tamoxifen to delete Tgfbr 2 encoding TGF- $\beta$ receptor 2 from all cells and tissues causes a significant decrease in alveolar macrophages [19]. In our study, Tgfb expression decreased with age and inhibition of TGF- $\beta$ reduced Cd206 expression in IVDs. Together with findings from previous studies, our results suggest that the age-related decrease in TGF- $\beta$ leads to a reduction in resident macrophages in IVDs.

The risk of IVD degeneration is thought to be greater in aging women than men $[29,30]$. A previous study showed that 17 -beta-estradiol promotes TGF- $\beta$ production in cartilaginous endplate cells derived from human IVDs [31]. Further, a recent study reported that estrogen deficiency alters the M1/M2 ratio in mouse bone marrow [32]. This evidence suggests that age-related changes in resident macrophages 


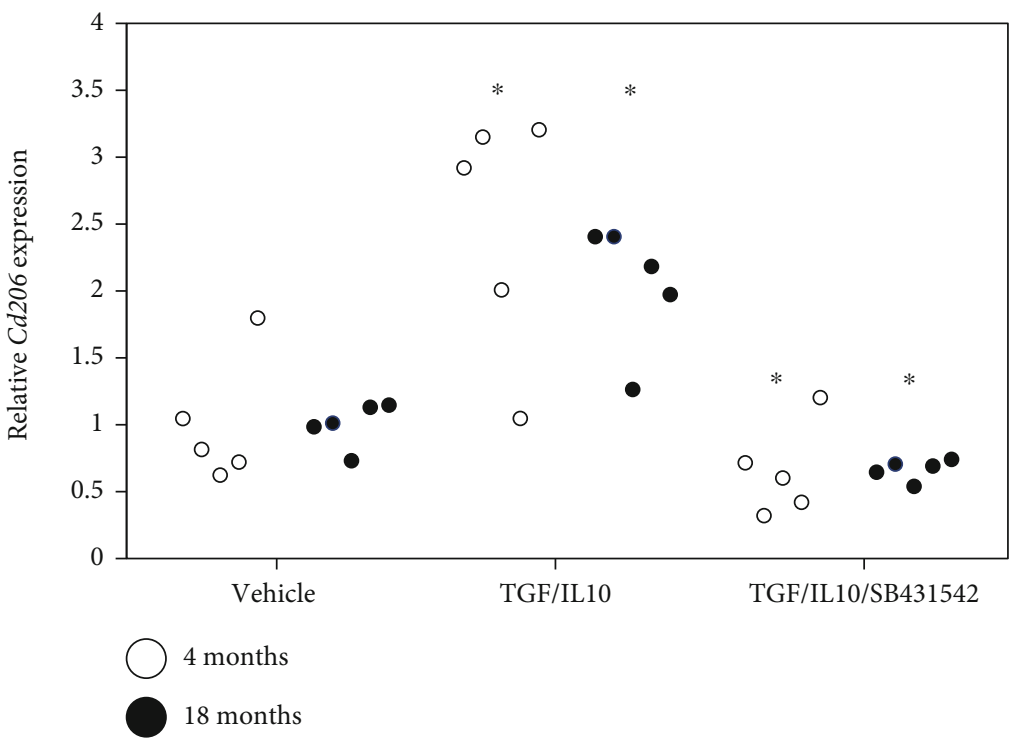

FIGURE 3: Effect of TGF- $\beta$ on M2 marker expression in vitro. Disc macrophages derived from young (age 4 months) and aged (age 18 months) mice were stimulated with $\alpha$-MEM (control), $10 \mathrm{ng} / \mathrm{ml}$ mouse recombinant (mr) TGF- $\beta+10 \mathrm{ng} / \mathrm{ml}$ IL- 10 (TGF/IL10), or $10 \mathrm{ng} / \mathrm{ml} \mathrm{mrTGF}$ $\beta+10 \mathrm{ng} / \mathrm{ml} \mathrm{IL}-10+10 \mu \mathrm{M}$ SB431542 (TGF/IL10/SB431542) for $24 \mathrm{~h}(n=5)$. Relative expression was determined based on expression in control samples. ${ }^{*} \mathrm{P}<0.05$ compared to vehicle.

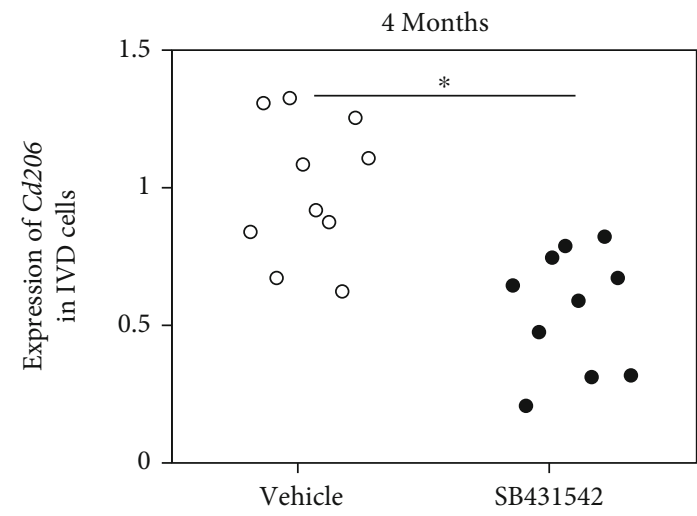

(a)

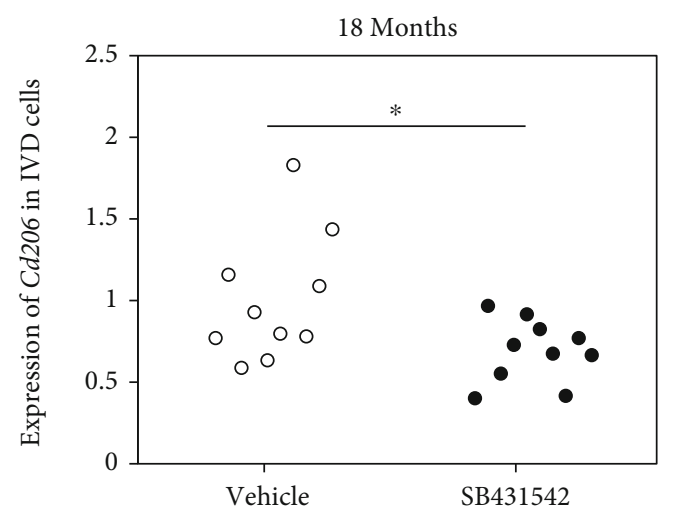

(b)

FIGURE 4: Effect of a TGF- $\beta$ inhibitor on $C d 206$ expression in vivo. Cd206 expression following administration of vehicle (DMSO) and TGF- $\beta$ inhibitor (SB431542) to (a) young and (b) aged mice ( $n=10$ for each group). ${ }^{*} P<0.05$ compared to vehicle.

may differ between male and female mice. Given that we only studied male mice, further investigations using female mice are needed.

There are several limitations in the present study. First, only male mice were used. Second, we did not separate NP and AF tissues for analysis. Third, how a reduction in resident macrophages is associated with IVD degeneration remains unclear. Finally, resident macrophages comprise a heterogeneous population. However, the roles of these individual populations remain unclear.

\section{Conclusions}

Resident macrophages decreased with age in IVDs, which may be associated with the concomitant decrease in TGF- $\beta$.
Our findings provide new insights into the mechanisms of age-related IVD pathology.

\section{Data Availability}

The data used to support the findings of this study are available from the corresponding author upon request.

\section{Conflicts of Interest}

The authors declare that there is no conflict of interest regarding the publication of this article.

\section{Authors' Contributions}

Yuji Yokozeki and Ayumu Kawakubo contributed equally to the work. 


\section{References}

[1] H. Yang, H. Liu, Z. Li et al., "Low back pain associated with lumbar disc herniation: role of moderately degenerative disc and annulus fibrous tears," International Journal of Clinical and Experimental Medicine, vol. 8, no. 2, pp. 1634-1644, 2015.

[2] C. J. Zheng and J. Chen, "Disc degeneration implies low back pain," Theoretical Biology and Medical Modelling, vol. 12, no. 1, p. 24, 2015.

[3] J. Antoniou, T. Steffen, F. Nelson et al., "The human lumbar intervertebral disc: evidence for changes in the biosynthesis and denaturation of the extracellular matrix with growth, maturation, ageing, and degeneration," Journal of Clinical Investigation, vol. 98, no. 4, pp. 996-1003, 1996.

[4] C. le Maitre, A. J. Freemont, and J. A. Hoyland, "Accelerated cellular senescence in degenerate intervertebral discs: a possible role in the pathogenesis of intervertebral disc degeneration," Arthritis Research \& Therapy, vol. 9, no. 3, p. R45, 2007.

[5] M. Molinos, C. R. Almeida, J. Caldeira, C. Cunha, R. M. Goncalves, and M. A. Barbosa, "Inflammation in intervertebral disc degeneration and regeneration," Journal of The Royal Society Interface, vol. 12, no. 108, article 20150429, 2015.

[6] M. J. Silva and N. Holguin, "Aging aggravates intervertebral disc degeneration by regulating transcription factors toward chondrogenesis," The FASEB Journal, vol. 34, no. 2, pp. 1970-1982, 2020.

[7] A. Kawakubo, K. Uchida, M. Miyagi et al., "Investigation of resident and recruited macrophages following disc injury in mice," Journal of Orthopaedic Research, vol. 38, no. 8, pp. 1703-1709, 2020.

[8] M. Miyagi, K. Uchida, S. Takano et al., "Role of CD14-positive cells in inflammatory cytokine and pain-related molecule expression in human degenerated intervertebral discs," Journal of Orthopaedic Research, 2020.

[9] M. Nakawaki, K. Uchida, M. Miyagi et al., "Sequential CCL2 expression profile after disc injury in mice," Journal of Orthopaedic Research, vol. 38, no. 4, pp. 895-901, 2020.

[10] K. R. Nakazawa, B. A. Walter, D. M. Laudier et al., "Accumulation and localization of macrophage phenotypes with human intervertebral disc degeneration," The Spine Journal, vol. 18, no. 2, pp. 343-356, 2018.

[11] M. Brigitte, C. Schilte, A. Plonquet et al., "Muscle resident macrophages control the immune cell reaction in a mouse model of notexin-induced myoinjury," Arthritis \& Rheumatism, vol. 62, no. 1, pp. 268-279, 2010.

[12] M. Guilliams, I. De Kleer, S. Henri et al., "Alveolar macrophages develop from fetal monocytes that differentiate into long-lived cells in the first week of life via GM-CSF," Journal of Experimental Medicine, vol. 210, no. 10, pp. 1977-1992, 2013.

[13] M. Nakawaki, K. Uchida, M. Miyagi et al., "Changes in nerve growth factor expression and macrophage phenotype following intervertebral disc injury in mice," Journal of Orthopaedic Research, vol. 37, no. 8, pp. 1798-1804, 2019.

[14] H. S. Schipper, B. Prakken, E. Kalkhoven, and M. Boes, “Adipose tissue-resident immune cells: key players in immunometabolism," Trends in Endocrinology \& Metabolism, vol. 23, no. 8, pp. 407-415, 2012.

[15] S. Watanabe, M. Alexander, A. V. Misharin, and G. R. S. Budinger, "The role of macrophages in the resolution of inflammation," Journal of Clinical Investigation, vol. 129, no. 7, pp. 2619-2628, 2019.
[16] A. R. Pinto, J. W. Godwin, A. Chandran et al., "Age-related changes in tissue macrophages precede cardiac functional impairment," Aging (Albany NY), vol. 6, no. 5, pp. 399-413, 2014.

[17] K. Tominaga and H. I. Suzuki, "TGF- $\beta$ signaling in cellular senescence and aging-related pathology," International Journal of Molecular Sciences, vol. 20, no. 20, p. 5002, 2019.

[18] S. Matsunaga, S. Nagano, T. Onishi, N. Morimoto, S. Suzuki, and S. Komiya, "Age-related changes in expression of transforming growth factor- $\beta$ and receptors in cells of intervertebral discs," Journal of Neurosurgery: Spine, vol. 98, no. 1, pp. 63-67, 2003.

[19] X. Yu, A. Buttgereit, I. Lelios et al., "The Cytokine TGF- $\beta$ Promotes the Development and Homeostasis of Alveolar Macrophages," Immunity, vol. 47, no. 5, pp. 903-912.e4, 2017.

[20] S. P. Zahner, J. M. Kel, C. A. Martina, I. Brouwers-Haspels, M. A. van Roon, and B. E. Clausen, "Conditional deletion of TGF- $\beta$ R1 using Langerin-Cre mice results in Langerhans cell deficiency and reduced contact hypersensitivity," The Journal of Immunology, vol. 187, no. 10, pp. 5069-5076, 2011.

[21] M. Miyagi, K. Uchida, S. Takano et al., "Macrophage-derived inflammatory cytokines regulate growth factors and painrelated molecules in mice with intervertebral disc injury," Journal of Orthopaedic Research ${ }^{\circledR}$, vol. 36, no. 8, pp. 2274-2279, 2018.

[22] S. Chen, S. Liu, K. Ma, L. Zhao, H. Lin, and Z. Shao, “TGF- $\beta$ signaling in intervertebral disc health and disease," Osteoarthritis and Cartilage, vol. 27, no. 8, pp. 1109-1117, 2019.

[23] D. R. Lemos, F. Babaeijandaghi, M. Low et al., "Nilotinib reduces muscle fibrosis in chronic muscle injury by promoting TNF- mediated apoptosis of fibro/adipogenic progenitors," Nature Medicine, vol. 21, no. 7, pp. 786-794, 2015.

[24] B. Z. Igyarto, M. C. Jenison, J. C. Dudda et al., "Langerhans cells suppress contact hypersensitivity responses via cognate CD4 interaction and langerhans cell-derived IL-10," Journal of Immunology, vol. 183, no. 8, pp. 5085-5093, 2009.

[25] Y. Shibata, P. Y. Berclaz, Z. C. Chroneos, M. Yoshida, J. A. Whitsett, and B. C. Trapnell, "GM-CSF regulates alveolar macrophage differentiation and innate immunity in the lung through PU.1," Immunity, vol. 15, no. 4, pp. 557-567, 2001.

[26] T. Suzuki, T. Sakagami, B. K. Rubin et al., "Familial pulmonary alveolar proteinosis caused by mutations in CSF2RA," Journal of Experimental Medicine, vol. 205, no. 12, pp. 2703-2710, 2008.

[27] K. Kambara, W. Ohashi, K. Tomita et al., "In Vivo Depletion of $\mathrm{CD}_{206}{ }^{+} \mathrm{M} 2 \mathrm{Macrophages} \mathrm{Exaggerates} \mathrm{Lung} \mathrm{Injury} \mathrm{in} \mathrm{Endo-}$ toxemic Mice," The American Journal of Pathology, vol. 185, no. 1, pp. 162-171, 2015.

[28] T. Hashimoto, T. Satoh, and H. Yokozeki, "Protective role of STAT6 in basophil-dependent prurigo-like allergic skin inflammation," The Journal of Immunology, vol. 194, no. 10, pp. 4631-4640, 2015.

[29] E. I. de Schepper, J. Damen, J. B. van Meurs et al., "The association between lumbar disc degeneration and low back pain: the influence of age, gender, and individual radiographic features," Spine (Phila Pa 1976), vol. 35, no. 5, pp. 531-536, 2010.

[30] Y. X. Wang, J. F. Griffith, X. J. Zeng et al., "Prevalence and sex difference of lumbar disc space narrowing in elderly Chinese men and women: osteoporotic fractures in men (Hong Kong) and osteoporotic fractures in women (Hong Kong) studies," Arthritis \& Rheumatism, vol. 65, no. 4, pp. 1004-1010, 2013. 
[31] B. Sheng, Y. Yuan, X. Liu et al., "Protective effect of estrogen against intervertebral disc degeneration is attenuated by miR221 through targeting estrogen receptor $\alpha$, Acta Biochimica et Biophysica Sinica, vol. 50, no. 4, pp. 345-354, 2018.

[32] C. Dou, N. Ding, C. Zhao et al., "Estrogen deficiency-mediated M2 macrophage osteoclastogenesis contributes to M1/M2 ratio alteration in ovariectomized osteoporotic mice," Journal of Bone and Mineral Research, vol. 33, no. 5, pp. 899-908, 2018. 